\title{
Ramsey numbers of path-matchings, covering designs, and 1-cores
}

\author{
Louis DeBiasio*† András Gyárfás ${ }^{\ddagger \S} \quad$ Gábor N. Sárközy ${ }^{\ddagger \uparrow||}$
}

October 1, 2020

\begin{abstract}
A path-matching of order $p$ is a vertex disjoint union of nontrivial paths spanning $p$ vertices. Burr and Roberts, and Faudree and Schelp determined the 2-color Ramsey number of path-matchings. In this paper we study the multicolor Ramsey number of path-matchings. Given positive integers $r, p_{1}, \ldots, p_{r}$, define $R^{P M}\left(p_{1}, \ldots, p_{r}\right)$ to be the smallest integer $n$ such that in any $r$-coloring of the edges of $K_{n}$ there exists a path-matching of color $i$ and order at least $p_{i}$ for some $i \in[r]$. Our main result is that for $r \geq 2$ and $p_{1} \geq \cdots \geq p_{r} \geq 2$, if $p_{1} \geq 2 r-2$, then

$$
R^{P M}\left(p_{1}, \ldots, p_{r}\right)=p_{1}-(r-1)+\sum_{i=2}^{r}\left\lceil\frac{p_{i}}{3}\right\rceil .
$$

Perhaps surprisingly, we show that when $p_{1}<2 r-2$, it is possible that $R^{P M}\left(p_{1}, \ldots, p_{r}\right)$ is larger than $p_{1}-(r-1)+\sum_{i=2}^{r}\left\lceil\frac{p_{i}}{3}\right\rceil$, but in any case we determine the correct value to within a constant (depending on $r$ ); i.e.

$$
p_{1}-(r-1)+\sum_{i=2}^{r}\left\lceil\frac{p_{i}}{3}\right\rceil \leq R^{P M}\left(p_{1}, \ldots, p_{r}\right) \leq\left\lceil p_{1}-\frac{r}{3}+\sum_{i=2}^{r} \frac{p_{i}}{3}\right\rceil .
$$

As a corollary we get that in every $r$-coloring of $K_{n}$ there is a monochromatic pathmatching of order at least $3\left\lfloor\frac{n}{r+2}\right\rfloor$, which is essentially best possible. We also determine $R^{P M}\left(p_{1}, \ldots, p_{r}\right)$ in all cases when the number of colors is at most 4 .

The proof of the main result uses a minimax theorem for path-matchings derived from a result of Las Vergnas (extending Tutte's 1-factor theorem) to show that the value of $R^{P M}\left(p_{1}, \ldots, p_{r}\right)$ depends on the block sizes in covering designs (which can be also formulated in terms of monochromatic 1-cores in colored complete graphs). While block sizes in covering designs have been studied intensively before, they seem to have only been studied in the uniform case (when all block sizes are equal). Then we obtain the result above by giving estimates on the block sizes in covering designs in the arbitrary (non-uniform) case.
\end{abstract}

\section{Introduction}

One of the seminal results in graph-Ramsey theory is the following theorem of Cockayne and Lorimer [4] which gives the $r$-color Ramsey number of a matching. Given positive integers $r, p_{1}, \ldots, p_{r}$ let $R^{M}\left(p_{1}, \ldots, p_{r}\right)$ be the smallest integer $n$ such that in every $r$-coloring of the edges of $K_{n}$, there exists a matching of color $i$ and order at least $p_{i}$ for some $i \in[r]$.

\footnotetext{
* Department of Mathematics, Miami University, Oxford, Ohio. debiasld@miamioh.edu

${ }^{\dagger}$ Research supported in part by Simons Foundation Collaboration Grant \#283194

¥Alfréd Rényi Institute of Mathematics, Hungarian Academy of Sciences, Budapest, P.O. Box 127, Budapest, Hungary, H-1364. gyarfas.andras@renyi.mta.hu, sarkozy.gabor@renyi.mta.hu

$\S$ Research supported in part by NKFIH Grant No. K116769.

๑ Computer Science Department, Worcester Polytechnic Institute, Worcester, MA.

"Research supported in part by NKFIH Grants No. K116769, K117879.
} 
Theorem 1.1 (Cockayne, Lorimer [4]). Let $r \geq 2$ and let $p_{1} \geq p_{2} \geq \cdots \geq p_{r} \geq 2$. Then

$$
R^{M}\left(p_{1}, \ldots, p_{r}\right)=p_{1}-(r-1)+\sum_{i=2}^{r}\left\lceil\frac{p_{i}}{2}\right\rceil .
$$

Theorem 1.1 is sharp, shown by the coloring $\left[p_{1}-1,\left\lceil\frac{p_{2}}{2}\right\rceil-1, \ldots,\left\lceil\frac{p_{r}}{2}\right\rceil-1\right]$ which is defined as follows: Given integers $r \geq 2$ and $t_{1}, \ldots, t_{r} \geq 0$, let $n=t_{1}+\cdots+t_{r}$ and define $\left[t_{1}, t_{2}, \ldots, t_{r}\right]$ to be the $r$-coloring of $K_{n}$ obtained by partitioning $V\left(K_{n}\right)$ as $\left\{A_{1}, \ldots, A_{r}\right\}$ such that $\left|A_{i}\right|=t_{i}$ for all $i \in[r]$, and coloring every edge $\{x, y\}$ with the maximum $j$ for which $\{x, y\}$ has a non-empty intersection with $A_{j}$.

We denote by $P_{k}$ the path with $k$ vertices and define a path-matching as a vertex disjoint union of paths, each with at least 2 vertices. The order of a path-matching $P$ is $|V(P)|$; i.e. the number of vertices spanned by $P$. A path-matching can clearly be written as vertex disjoint union of $P_{2}$-s and $P_{3}$-s. Thus the maximum order of a path-matching in a graph is equal to the maximum order of a path-matching containing only $P_{2}$ and $P_{3}$ components. We note that sometimes a path-matching is called a linear forest in the literature [3, 6].

Here we study the Ramsey problem for path-matchings: what is the order of the largest monochromatic path-matching we can find in every $r$-coloring of the edges of $K_{n}$ ? Note that this belongs to the part of Ramsey theory where the target graph is a large monochromatic member of a family instead of a specified graph. Many other families have been investigated, for example the family of connected graphs, graphs without isolated vertices, highly connected graphs, graphs of small diameter, etc. A survey on problems of this flavor is $[9]$.

Given positive integers $r, p_{1}, \ldots, p_{r}$, define $R^{P M}\left(p_{1}, \ldots, p_{r}\right)$ to be the smallest integer $n$ such that in any $r$-coloring of the edges of $K_{n}$ there exists a path-matching of color $i$ and order at least $p_{i}$ for some $i \in[r]$. If $p_{1}=\cdots=p_{r}=p$, we sometimes write $R_{r}^{P M}(p)$ instead of $R^{P M}(p, \ldots, p)$.

Burr and Roberts [3] proved that for all integers $p \geq 2, R^{P M}(p, p)=\left\lceil\frac{4 p}{3}\right\rceil-1$. Later, Faudree and Schelp [6] proved a non-symmetric version; that is, for all integers $p_{1} \geq p_{2} \geq 2$,

$$
R^{P M}\left(p_{1}, p_{2}\right)=p_{1}+\left\lceil\frac{p_{2}}{3}\right\rceil-1
$$

(In fact, in both cases above the authors prove a stronger statement where the formula takes into account the number of paths of odd length.) We extend these results to $r$-colorings with $r \geq 3$.

Our main result is that we completely determine $R^{P M}$ provided $p_{1}$ is not too small compared to $r$. Note the similarity between Theorem 1.1 and Theorem [1.2.

Theorem 1.2. Let $r \geq 3$ and let $p_{1} \geq p_{2} \geq \cdots \geq p_{r} \geq 2$ be integers with $p_{1} \geq 4$. If $p_{1} \geq$ $2 r-3-\sum_{i=2}^{r} 3\left(\left\lceil\frac{p_{i}}{3}\right\rceil-\frac{p_{i}}{3}\right)$, then

$$
R^{P M}\left(p_{1}, \ldots, p_{r}\right)=p_{1}-(r-1)+\sum_{i=2}^{r}\left\lceil\frac{p_{i}}{3}\right\rceil .
$$

The lower bound in Theorem 1.2 comes from the extremal coloring

$$
\left[p_{1}-1,\left\lceil\frac{p_{2}}{3}\right\rceil-1, \ldots,\left\lceil\frac{p_{r}}{3}\right\rceil-1\right] .
$$

Note that if at least $r-3$ of the terms $p_{2}, \ldots, p_{r}$ were congruent to $1 \bmod 3$, then $p_{1} \geq$ $2 r-3-\sum_{i=2}^{r} 3\left(\left\lceil\frac{p_{i}}{3}\right\rceil-\frac{p_{i}}{3}\right)$ reduces to $p_{1} \geq 3$ and thus we have an exact result with no extra conditions.

It would be natural to guess that the requirement that $p_{1}$ be sufficiently large in terms of $r$ is unnecessary. However, in Corollary[3.5, we will prove that if say $\frac{3}{2}\left\lfloor\frac{\sqrt{8 r+1}+1}{2}\right\rfloor>p_{1} \geq \cdots \geq p_{r} \geq 3$ with all $p_{i}$ being divisible by 3 , then, perhaps surprisingly,

$$
R^{P M}\left(p_{1}, \ldots, p_{r}\right)>p_{1}-(r-1)+\sum_{i=2}^{r}\left\lceil\frac{p_{i}}{3}\right\rceil .
$$


Thus the complete determination of $R^{P M}\left(p_{1}, \ldots, p_{r}\right)$ is still open and as we will see later, determining $R^{P M}\left(p_{1}, \ldots, p_{r}\right)$ when $p_{1}$ is fixed and $r$ is large may be difficult because of the connection with covering designs.

However, our next main result shows that in any case we can determine $R^{P M}\left(p_{1}, \ldots, p_{r}\right)$ to within a constant (depending on $r$ ).

Theorem 1.3. Let $r \geq 2$ and let $p_{1} \geq p_{2} \geq \cdots \geq p_{r} \geq 2$ be integers. Then

$$
R^{P M}\left(p_{1}, \ldots, p_{r}\right) \leq\left\lceil p_{1}-\frac{r}{3}+\sum_{i=2}^{r} \frac{p_{i}}{3}\right\rceil \text {. }
$$

We get the following corollary of Theorem 1.3 in the case where all the $p_{i}$-s are equal (stated here using the inverse formulation).

Corollary 1.4. Let $r \geq 2$ be an integer. Every $r$-coloring of $K_{n}$ contains a monochromatic path-matching of order at least $3\left\lfloor\frac{n}{r+2}\right\rfloor$.

This is sharp if $n$ is divisible by $r+2$ as shown by the extremal coloring $\left[\frac{3 n}{r+2}, \frac{n}{r+2}, \ldots, \frac{n}{r+2}\right]$.

We note that establishing Theorem 1.2 requires a bit more technical work than Theorem 1.3. however, since Theorem 1.2 is tight in many more cases than Theorem [1.3, it is worth it. However, if one was only interested in Corollary [1.4 Theorem 1.3 would suffice.

\section{Covering designs, 1-cores, and a deficiency formula for path-matchings}

The proof of Theorems 1.2 and 1.3 are based on a minimax theorem on path-matchings derived from a result of Las Vergnas (which provides an analogue of Tutte's 1-factor theorem for pathmatchings). Interestingly, when we apply this minimax theorem to $r$-colored complete graphs, we need a suitable estimate on block sizes in covering designs (which can be also formulated as an estimate on the sizes of 1-cores in colored complete graphs).

We describe all of this in detail in the following subsections.

\subsection{Ramsey numbers of covering designs}

A covering design is a family of sets called blocks in an $n$-element set $V$ such that each pair of $V$ is covered by at least one block. If all blocks have the same size $p$, then $C(n, p)$ is used to denote the minimum number of blocks in a covering design. The asymptotics of $C(n, p)$ for fixed $p$ was determined by Erdős and Hanani [5] and the breakthrough of R. M. Wilson [18] provided equality with constructing block designs for every admissible $n \geq n_{0}(p)$.

One can formulate the inverse problem of finding $C(n, p)$ as a Ramsey problem. For given $r, p$ find the smallest $n=R_{r}(p)$ such that every covering design on $n$ vertices with $r$ blocks must contain a block of size at least $p$. Mills [13] determined the asymptotic of $R_{r}(p) / p$ for $r \leq 13$ and this ratio is also known for values of $r$ in the form $q^{2}+q+1$ or $q^{2}+q$ when $P G(2, q)$ exists (see the excellent survey of Füredi [7, Chapter 7]). This problem was also studied, using a different formulation by Horák and Sauer [10]. However, there is no conjecture for the limit of $R_{r}(p) / p$ for general $r$.

For our goals we consider covering designs with variable block sizes, which leads to the offdiagonal case of the Ramsey number $R_{r}(p)$. In the next section we will obtain estimates for this Ramsey number.

\subsection{Ramsey numbers of 1-cores}

The Ramsey number of covering designs can be reformulated in graph theoretic language as the Ramsey number of graphs with minimum degree at least one, i.e. graphs without isolated 
vertices. With a slight abuse of the original definition, we say that $G$ is a 1-core if $G$ has minimum degree at least one. (The $k$-core of a graph $G$ was defined by Seidman [17] as the largest connected subgraph of $G$ with minimum degree at least $k$, subsequently many papers [1] and textbooks [2] define it without the connectivity condition.) To see that the Ramsey number of the family of 1 -cores is the same as the Ramsey number of a covering design, given an $r$-coloring of $K_{n}$, we can replace the 1-core of color $i$ with a clique of color $i$ (allowing for edges to have multiple colors) without changing the size of the 1-core and thus each clique corresponds to a block in the covering design language.

Given positive integers $r, p_{1}, \ldots, p_{r}$, let $R^{1 C}\left(p_{1}, \ldots, p_{r}\right)$ be the smallest integer $n$ such that in every $r$ coloring of the edges of $K_{n}$, there exists a 1-core of color $i$ and order at least $p_{i}$ for some $i \in[r]$. Equivalently, $R^{1 C}\left(p_{1}, \ldots, p_{r}\right)$ is the smallest integer $n$ such that the edges of $K_{n}$ cannot be covered with cliques of order $p_{1}-1, \ldots, p_{r}-1$. If $p_{1}=\cdots=p_{r}=p$, we write $R_{r}^{1 C}(p)$ instead of $R^{1 C}(p, \ldots, p)$. We also note that Observation 2.1 and Proposition 2.2 apply with $R^{1 C}$ in place of $R^{P M}$.

First note the following which essentially means that we can assume $p_{i} \geq 3$ for all $i \in[r]$.

Observation 2.1. For all integers $r \geq 2$ and $p_{1} \geq p_{2} \geq \cdots \geq p_{r} \geq 2, R^{P M}\left(p_{1}, \ldots, p_{r}\right)=$ $R^{P M}\left(p_{1}, \ldots, p_{r}, 2\right)=R^{P M}\left(p_{1}, \ldots, p_{r}, 1\right)$ and similarly for $R^{1 C}$.

Next we have the following result when $p_{1}=\cdots=p_{r}=3$.

Proposition 2.2. Let $r \geq 2$ be an integer. Then $R_{r}^{P M}(3)=R_{r}^{1 C}(3)$ is the smallest integer $n$ such that $\left(\begin{array}{l}n \\ 2\end{array}\right)>r$. In other words, $R_{r}^{P M}(3)=R_{r}^{1 C}(3)=\left\lfloor\frac{\sqrt{8 r+1}+1}{2}\right\rfloor+1$.

Proof. If $\left(\begin{array}{l}n \\ 2\end{array}\right)>r$, then in every $r$-coloring of $K_{n}$, some color must be used more than once. If $\left(\begin{array}{l}n \\ 2\end{array}\right) \leq r$, then there exists an $r$-coloring of $K_{n}$ where each color is used at most once.

We now give estimates on $R^{1 C}\left(p_{1}, \ldots, p_{r}\right)$ where the focus is on the off-diagonal case, which to the best of our knowledge has not been studied.

In the language of covering designs, we have $R_{r}^{1 C}(p) \leq n$ if and only if $C(n, p-1)>r$. So the vast literature on covering designs gives upper bounds on $R_{r}^{1 C}(p)$. A simple lower bound given by Erdős and Hanani [5] is $C(v, p-1) \geq\left(\begin{array}{c}v \\ 2\end{array}\right) /\left(\begin{array}{c}p-1 \\ 2\end{array}\right)=\frac{v(v-1)}{(p-1)(p-2)}$. A more refined lower bound is the so-called Schönheim bound [15], which says $C(v, p-1) \geq\left\lceil\frac{v}{p-1}\left\lceil\frac{v-1}{p-2}\right\rceil\right\rceil$.

This first Observation is just a generalization of the Erdős-Hanani lower bound in the nonuniform case. It says that if the total number of edges in cliques of orders $p_{1}-1, \ldots, p_{r}-1$ respectively is less than the number of edges in $K_{n}$, then it is not possible to cover $K_{n}$ with those cliques. While this Observation is almost trivial, there are situations where this is the best estimate to use.

Observation 2.3. Let $n, r \geq 2$ and let $p_{1} \geq p_{2} \geq \cdots \geq p_{r} \geq 2$ be integers. If

$$
\sum_{i=1}^{r}\left(\begin{array}{c}
p_{i}-1 \\
2
\end{array}\right)<\left(\begin{array}{l}
n \\
2
\end{array}\right),
$$

then $R^{1 C}\left(p_{1}, \ldots, p_{r}\right) \leq n$.

The next bound is essentially a non-uniform generalization of results in [10] and [13. Again there are situations where this next bound is the best estimate to use. This result can be seen to be tight when a projective plane of order $q$ exists, $\ell \in\{0,1\}$ and $p_{1}=\cdots=p_{r}=\left\lceil\frac{(q+1) n}{q^{2}+q+\ell}\right\rceil$.

Proposition 2.4. Let $n, r \geq 2$ and let $p_{1} \geq p_{2} \geq \cdots \geq p_{r} \geq 2$ be integers. If there exists an integer $1 \leq t \leq r-1$ such that

$$
p_{1} \leq\left\lceil\frac{n+t-1}{t}\right\rceil \text { and } \sum_{i=1}^{r}\left(p_{i}-1\right)<(t+1) n
$$

then $R^{1 C}\left(p_{1}, \ldots, p_{r}\right) \leq n$. 
We say that a vertex sees a color if it is incident with an edge of that color.

Proof. Suppose there exists an integer $1 \leq t \leq r-1$ such that $p_{1} \leq\left\lceil\frac{n+t-1}{t}\right\rceil$ and $\sum_{i=1}^{r}\left(p_{i}-1\right)<$ $(t+1) n$ and consider an $r$-edge coloring of a complete graph on a set $V$ of vertices where $|V|=n$. For all $i \in[r]$, let $S_{i}$ be the 1-core of color $i$ and suppose for contradiction that $\left|S_{i}\right| \leq p_{i}-1$ for all $i \in[r]$. So for all $i \in[r]$, there exists at least $n-p_{i}+1$ vertices which do not see color $i$.

So on average, the number of colors a vertex does not see is at least

$$
\frac{\sum_{i=1}^{r}\left(n-p_{i}+1\right)}{n}=r-\frac{\sum_{i=1}^{r}\left(p_{i}-1\right)}{n}>r-(t+1) .
$$

This implies some vertex $v$ sees at most $t$ colors. So $v$ is contained in a monochromatic 1-core of order at least $1+\left\lceil\frac{n-1}{t}\right\rceil=\left\lceil\frac{n+t-1}{t}\right\rceil$ contradicting the original assumption.

Now we come to our main result of this section. We note that this result can be seen to be tight in certain cases, such as when either of the first two terms are the maximum.

Theorem 2.5. Let $r \geq 2$ and let $p_{1} \geq p_{2} \geq \cdots \geq p_{r} \geq 2$ be integers. Then

$$
R^{1 C}\left(p_{1}, \ldots, p_{r}\right) \leq \max \left\{p_{1},\left\lceil\frac{p_{1}+p_{2}+p_{3}}{2}\right\rceil-1,\left\lceil\frac{p_{1}}{3}-\frac{r}{3}+\sum_{i=1}^{r} \frac{p_{i}}{3}\right\rceil\right\} .
$$

Proof. Consider an $r$-edge coloring of a complete graph on a set $V$ of vertices where

$$
|V|=n=\max \left\{p_{1},\left\lceil\frac{p_{1}+p_{2}+p_{3}}{2}\right\rceil-1,\left\lceil\frac{p_{1}}{3}-\frac{r}{3}+\sum_{i=1}^{r} \frac{p_{i}}{3}\right\rceil\right\} .
$$

For all $i \in[r]$, let $S_{i}$ be the 1-core of color $i$ and suppose for contradiction that $\left|S_{i}\right| \leq p_{i}-1$ for all $i \in[r]$.

Consider the partition $V=X_{1} \cup X_{2} \cup X_{3}$, where for $i \in[2], X_{i}$ is the set of vertices which are incident with edges of exactly $i$ different colors and $X_{3}$ is the set of vertices incident with edges of at least 3 different colors. Note that every vertex is incident with edges of at least two different colors (i.e. $X_{1}=\emptyset$ ); otherwise there is a monochromatic 1-core on $n$ vertices, but by (3) and the indirect assumption, we have $p_{1} \leq n \leq p_{1}-1$, a contradiction.

Note that

$$
|V|=n \leq \sum_{i=1}^{r}\left|S_{i}\right|-\left|X_{2}\right|-2\left|X_{3}\right|
$$

since the vertices in $X_{2}$ are counted twice in the sum $\sum_{i=1}^{r}\left|S_{i}\right|$, and the vertices in $X_{3}$ are counted at least three times in the $\operatorname{sum} \sum_{i=1}^{r}\left|S_{i}\right|$.

Claim 1. $\left|X_{2}\right| \leq p_{1}-1$.

Proof. Since the graph induced by $X_{2}$ is locally 2-colored (i.e. each vertex sees at most two colors), either there exists a color $i$ such that every vertex in $X_{2}$ sees color $i$, in which case by the indirect assumption, $\left|X_{2}\right| \leq p_{i}-1 \leq p_{1}-1$, or there are a total of at most three colors used on $X_{2}$. We now show that the latter is impossible (because of the choice of $n$ ).

Suppose there is no color seen by every vertex in $X_{2}$ and let $i, j, k$ be the three colors used on $X_{2}$. In this case, it can be easily seen that $X_{2}$ contains a set of three vertices $\{a, b, c\}$ such that $a b$ has color $i, a c$ has color $j$ and $b c$ has color $k$. Since every edge incident with $a$ has color $i$ or $j$, every edge incident with $b$ has color $i$ or $k$, and every edge incident with $c$ has color $j$ or $k$, it is the case that for all $v \in V \backslash\{a, b, c\}, v$ sends at least two of the colors $i, j, k$ to $\{a, b, c\}$. So, regardless of whether $X_{3}=\emptyset$ or not, every vertex in $V$ sees at least two of the colors $i, j, k$ and thus

$$
2 n \leq\left|S_{i}\right|+\left|S_{j}\right|+\left|S_{k}\right| \leq\left|S_{1}\right|+\left|S_{2}\right|+\left|S_{3}\right| \leq p_{1}+p_{2}+p_{3}-3,
$$

contradicting the choice of $n$. 
Now by Claim 1 we have

$$
\left|X_{3}\right|=n-\left|X_{2}\right| \geq n-p_{1}+1 .
$$

Then from (4) and (5) we get

$$
n \leq \sum_{i=1}^{r}\left|S_{i}\right|-\left|X_{2}\right|-2\left|X_{3}\right| \leq \sum_{i=1}^{r} p_{i}-n-\left|X_{3}\right|-r \leq \sum_{i=1}^{r} p_{i}-n-\left(n-p_{1}+1\right)-r
$$

which, by (3), implies

$$
3\left\lceil\frac{p_{1}}{3}-\frac{r}{3}+\sum_{i=1}^{r} \frac{p_{i}}{3}\right\rceil \leq 3 n \leq p_{1}-(r+1)+\sum_{i=1}^{r} p_{i},
$$

a contradiction.

\section{Large monochromatic path-matchings}

\subsection{Deficiency formula for path-matchings}

The deficiency formula for path-matchings can be derived from a special case of a result of Las Vergnas [11. Recall that a path-matching can always be written as vertex disjoint union of $P_{2}$-s and $P_{3}$-s, so the maximum order of a path-matching in a graph is equal to the maximum order of a path-matching containing only $P_{2}$ and $P_{3}$ components. Let $f, g$ be integer-valued functions on the vertex set $V$ of a graph $G$ such that $0 \leq g(v) \leq 1 \leq f(v)$ for all $v \in V$. A $(g, f)$-factor is a subgraph $F$ of $G$ satisfying $g(v) \leq d_{F}(v) \leq f(v)$ for all $v \in V$. Las Vergnas [11 gave a necessary and sufficient condition for the existence of a $(g, f)$ factor of a graph. If $g \equiv 1, f \equiv 2$ then the existence of a $(g, f)$-factor is equivalent to the existence of a perfect path-matching; that is, a path-matching covering all vertices of $G$. In this case the condition simplifies and can be stated as follows. Let $q_{G}(S)$ denote the number of isolated vertices of a graph $G$ in a set $S \subset V(G)$.

Theorem 3.1 (Las Vergnas [11]). There exists a perfect path-matching in $G$ if and only if $2|X| \geq q_{G}(V(G) \backslash X)$ for all $X \subset V(G)$.

This result is "self-refining" in the sense that one can easily derive from it the minimax formula for the deficiency of path-matchings (see [12, Exercise 3.1.16] in which Berge's formula is derived from Tutte's theorem). Let $p d(G)$ be the path-matching deficiency of $G$, the number of vertices uncovered by any path-matching of maximum order in $G$.

Corollary 3.2. $p d(G)=\max \left\{q_{G}(V(G) \backslash X)-2|X|: X \subset V(G)\right\}$.

We call a set $X$ achieving the maximum in Corollary 3.2 an $L V$ set.

\subsection{1-cores and path-matchings}

Our main general result shows that the Ramsey numbers for path-matchings are tied to the Ramsey numbers for 1-cores in a fundamental way. First, given positive integers $r \geq 2, p_{1} \geq$ $p_{2} \geq \cdots \geq p_{r} \geq 2$, and $d$, let

$$
f^{d}\left(p_{1}, \ldots, p_{r}\right)=\max \left\{R^{1 C}\left(p_{1}-d x_{1}, \ldots, p_{r}-d x_{r}\right)+\sum_{i=1}^{r} x_{i}: 0 \leq x_{i}<\frac{p_{i}}{d} \text { for all } i \in[r]\right\},
$$

where the $x_{i}$ 's are integers.

Theorem 3.3. Let $r \geq 2$ and let $p_{1} \geq p_{2} \geq \cdots \geq p_{r} \geq 2$ be integers. Then

$$
R^{P M}\left(p_{1}, \ldots, p_{r}\right)=f^{3}\left(p_{1}, \ldots, p_{r}\right) .
$$


For instance this result says $R^{P M}(6,6,6,6,6)=\max \left\{R^{1 C}(6,6,6,6,6), R^{1 C}(6,6,6,6,3)+\right.$ $\left.1, R^{1 C}(6,6,6,3,3)+2, R^{1 C}(6,6,3,3,3)+3, R^{1 C}(6,3,3,3,3)+4, R^{1 C}(3,3,3,3,3)+5\right\}$.

The following example provides the lower bound in Theorem 3.3 .

Example 3.4. Let $r, p_{1}, \ldots, p_{r}, x_{1}, \ldots, x_{r}$ be integers with $r \geq 2, p_{1} \geq p_{2} \geq \cdots \geq p_{r} \geq 2$, and for all $i \in[r], 0 \leq x_{i}<\frac{p_{i}}{3}$. If $n=R^{1 C}\left(p_{1}-3 x_{1}, \ldots, p_{r}-3 x_{r}\right)+\sum_{i=1}^{r} x_{i}$, then there exists an $r$-coloring of $K_{n-1}$ such that for all $i \in[r]$, the largest path-matching of color $i$ has order at most $p_{i}-1$.

Proof. Set $t=R^{1 C}\left(p_{1}-3 x_{1}, \ldots, p_{r}-3 x_{r}\right)$ and start with an $r$-coloring of $K_{t-1}$ such that for all $i \in[r]$, the largest 1-core of color $i$ has order at most $p_{i}-3 x_{i}-1$ which must exist by the definition of $R^{1 C}\left(p_{1}-3 x_{1}, \ldots, p_{r}-3 x_{r}\right)$. For all $i \in[r]$, add a set $X_{i}$ of vertices such that $\left|X_{i}\right|=x_{i}$ and color all edges incident with $X_{i}$ with color $i$. This gives a coloring of $K_{n-1}$ such that for all $i \in[r]$, the largest path-matching of color $i$ has order at most $p_{i}-3 x_{i}-1+3 x_{i}=p_{i}-1$ (by Corollary 3.2 for instance).

The following proof is inspired by Petrov's [14] non-inductive proof of Theorem 1.1 (for another similar proof see [19]).

Proof of Theorem 3.3. The lower bound follows from Example 3.4

For the upper bound, consider an $r$-edge coloring of a complete graph on a vertex set $V$ with $|V|=n=f^{3}\left(p_{1}, \ldots, p_{r}\right)$.

For all $i \in[r]$, let $G_{i}$ the subgraph induced by the edges of color $i$. Suppose, for contradiction, that for all $i \in[r]$ the largest path-matching in $G_{i}$ has order at most $p_{i}-1$. For all $i \in[r]$, apply Corollary 3.2 to get an LV set $X_{i}$ and corresponding independent set $S_{i}$ such that

$$
\left|S_{i}\right| \geq 2\left|X_{i}\right|+n-\left(p_{i}-1\right),
$$

and note that since $\left|S_{i}\right| \leq n-\left|X_{i}\right|$, we have

$$
0 \leq\left|X_{i}\right|<\frac{p_{i}}{3}
$$

Let $X=\cup_{i=1}^{r} X_{i}$ and let $Y=V \backslash X$. For all $i \in[r]$ we have

$$
\left|S_{i} \cap Y\right| \geq\left|S_{i}\right|-\left(|X|-\left|X_{i}\right|\right) \geq 2\left|X_{i}\right|+|V|-\left(p_{i}-1\right)-\left(|X|-\left|X_{i}\right|\right)=|Y|-\left(p_{i}-3\left|X_{i}\right|-1\right) .
$$

In other words, the largest 1-core of color $i$ in the graph $G_{i}[Y]$ has order at most $p_{i}-3\left|X_{i}\right|-1$.

We have by the definition of $n$,

$$
|Y|=n-|X| \geq n-\sum_{i=1}^{r}\left|X_{i}\right| \geq R^{1 C}\left(p_{1}-3\left|X_{1}\right|, \ldots, p_{r}-3\left|X_{r}\right|\right)
$$

so $Y$ contains a 1-core of color $i$ and order at least $p_{i}-3\left|X_{i}\right|$ for some $i \in[r]$, contradicting our original assumption.

Now we obtain lower bounds on $R^{P M}\left(p_{1}, \ldots, p_{r}\right)$ as a corollary of Theorem 3.3 .

Corollary 3.5. Let $r, p_{1}, \ldots, p_{r}$ be integers with $r \geq 2, p_{1} \geq p_{2} \geq \cdots \geq p_{r} \geq 2$ and let $s$ be the number of $p_{i}$ 's which are divisible by 3. Then the following hold:

(i) $R^{P M}\left(p_{1}, \ldots, p_{r}\right) \geq p_{1}-(r-1)+\sum_{i=2}^{r}\left\lceil\frac{p_{i}}{3}\right\rceil$,

(ii) $R^{P M}\left(p_{1}, \ldots, p_{r}\right) \geq R_{s}^{1 C}(3)+\sum_{i=1}^{r}\left(\left\lceil\frac{p_{i}}{3}\right\rceil-1\right)=\left\lfloor\frac{\sqrt{8 s+1}+1}{2}\right\rfloor+1+\sum_{i=1}^{r}\left(\left\lceil\frac{p_{i}}{3}\right\rceil-1\right)$.

Note that under certain circumstances, such as all $p_{i}$ being divisible by 3 and $p_{1}<\frac{3}{2}\left\lfloor\frac{\sqrt{8 r+1}+1}{2}\right\rfloor$, we have

$$
\left\lfloor\frac{\sqrt{8 r+1}+1}{2}\right\rfloor+1+\sum_{i=1}^{r}\left(\left\lceil\frac{p_{i}}{3}\right\rceil-1\right)>p_{1}-(r-1)+\sum_{i=2}^{r}\left\lceil\frac{p_{i}}{3}\right\rceil .
$$

For instance, we have $R_{10}^{P M}(6)>6-(10-1)+(10-1) \cdot 2=15$. 
Proof. (i) This follows from the fact that

$$
\begin{aligned}
& \max \left\{R^{1 C}\left(p_{1}-3 x_{1}, \ldots, p_{r}-3 x_{r}\right)+\sum_{i=1}^{r} x_{i}: 0 \leq x_{i}<\frac{p_{i}}{3} \text { for all } i \in[r]\right\} \\
& \geq R^{1 C}\left(p_{1}, p_{2}-3\left(\left\lceil\frac{p_{2}}{3}\right\rceil-1\right), \ldots, p_{r}-3\left(\left\lceil\frac{p_{r}}{3}\right\rceil-1\right)\right)+\sum_{i=2}^{r}\left(\left\lceil\frac{p_{i}}{3}\right\rceil-1\right) \geq p_{1}+\sum_{i=2}^{r}\left(\left\lceil\frac{p_{i}}{3}\right\rceil-1\right),
\end{aligned}
$$

where the last inequality holds since in general we have $R^{1 C}\left(a_{1}, \ldots, a_{r}\right) \geq a_{1}$ for all $a_{1} \geq \cdots \geq a_{r}$.

(ii) This follows from the fact that

$$
\begin{aligned}
& \max \left\{R^{1 C}\left(p_{1}-3 x_{1}, \ldots, p_{r}-3 x_{r}\right)+\sum_{i=1}^{r} x_{i}: 0 \leq x_{i}<\frac{p_{i}}{3} \text { for all } i \in[r]\right\} \\
& \geq R^{1 C}\left(p_{1}-3\left(\left\lceil\frac{p_{1}}{3}\right\rceil-1\right), p_{2}-3\left(\left\lceil\frac{p_{2}}{3}\right\rceil-1\right), \ldots, p_{r}-3\left(\left\lceil\frac{p_{r}}{3}\right\rceil-1\right)\right)+\sum_{i=1}^{r}\left(\left\lceil\frac{p_{i}}{3}\right\rceil-1\right) \\
& =R_{s}^{1 C}(3)+\sum_{i=1}^{r}\left(\left\lceil\frac{p_{i}}{3}\right\rceil-1\right)=\left\lfloor\frac{\sqrt{8 s+1}+1}{2}\right\rfloor+1+\sum_{i=1}^{r}\left(\left\lceil\frac{p_{i}}{3}\right\rceil-1\right)
\end{aligned}
$$

where the last equality holds by Proposition 2.2

Now we show how Theorem 3.3 implies the result for two colors (which follows from the result of Faudree and Schelp [6]). However, the proof of their result is lengthy and relies on the 2-color Ramsey number of paths determined in [8]. Therefore the short proof below is perhaps of some interest.

Note that since a graph or its complement is connected, we clearly have

$$
R^{1 C}\left(p_{1}, p_{2}\right)=\max \left\{p_{1}, p_{2}\right\}
$$

Corollary 3.6. Let $p_{1} \geq p_{2} \geq 2$ be integers. Then

$$
R^{P M}\left(p_{1}, p_{2}\right)=p_{1}+\left\lceil\frac{p_{2}}{3}\right\rceil-1 .
$$

Proof. By (8), we have for all $0 \leq x_{1}<\frac{p_{1}}{3}, 0 \leq x_{2}<\frac{p_{2}}{3}$,

$R^{1 C}\left(p_{1}-3 x_{1}, p_{2}-3 x_{2}\right)+x_{1}+x_{2} \leq \max \left\{p_{1}-3 x_{1}, p_{2}-3 x_{2}\right\}+x_{1}+x_{2} \leq p_{1}+x_{2} \leq p_{1}+\left\lceil\frac{p_{2}}{3}\right\rceil-1$.

Thus by Theorem 3.3 we have $R^{P M}\left(p_{1}, p_{2}\right)=f^{3}\left(p_{1}, p_{2}\right) \leq p_{1}+\left\lceil\frac{p_{2}}{3}\right\rceil-1$.

\subsection{The proofs of Theorem 1.2 and Theorem 1.3}

We obtain the proofs by combining using Theorem 2.5 to get an appropriate upper bound on $f^{3}\left(p_{1}, \ldots, p_{r}\right)$ at which point the result follows from Theorem 3.3 So the proof just reduces to checking some technical inequalities which we first collect here.

Fact 3.7. Let $r \geq 3$ and let $a_{1} \geq a_{2} \geq \cdots \geq a_{r} \geq 2$ be integers with $a_{1} \geq 3$.

(i) $\left\lceil\frac{2 a_{1}}{3}-\frac{r}{3}+\sum_{i=1}^{r} \frac{a_{i}}{3}\right\rceil \geq\left\lceil\frac{a_{1}+a_{2}+a_{3}}{2}\right\rceil-1$

(ii) $a_{1}-(r-1)+\sum_{i=2}^{r}\left\lceil\frac{a_{i}}{3}\right\rceil \geq\left\lceil\frac{a_{1}}{3}-\frac{r}{3}+\sum_{i=1}^{r} \frac{a_{i}}{3}\right\rceil$ if and only if $a_{1} \geq 2 r-3-\sum_{i=2}^{r} 3\left(\left\lceil\frac{a_{i}}{3}\right\rceil-\frac{a_{i}}{3}\right)$.

(iii) $a_{1}-(r-1)+\sum_{i=2}^{r}\left\lceil\frac{a_{i}}{3}\right\rceil \geq\left\lceil\frac{a_{1}+a_{2}+a_{3}}{2}\right\rceil-1+\sum_{i=4}^{r}\left(\left\lceil\frac{a_{i}}{3}\right\rceil-1\right)$ if and only if $a_{1} \geq 2+$ $\left(a_{2}-2\left\lceil\frac{a_{2}}{3}\right\rceil\right)+\left(a_{3}-2\left\lceil\frac{a_{3}}{3}\right\rceil\right)$. In particular, this holds when $a_{1} \geq 4$. 
Proof. (i) First note that $\left\lceil\frac{2 a_{1}}{3}-\frac{r}{3}+\sum_{i=1}^{r} \frac{a_{i}}{3}\right\rceil \geq a_{1}+\frac{a_{2}}{3}+\frac{a_{3}}{3}-1$ and since $a_{1} \geq 3$, we have $a_{1}+\frac{a_{2}}{3}+\frac{a_{3}}{3}-1 \geq \frac{a_{1}+a_{2}+a_{3}-1}{2} \geq\left\lceil\frac{a_{1}+a_{2}+a_{3}}{2}\right\rceil-1$

(ii) Since $a_{1}-(r-1)+\sum_{i=2}^{r}\left\lceil\frac{a_{i}}{3}\right\rceil$ is an integer, $a_{1}-(r-1)+\sum_{i=2}^{r}\left\lceil\frac{a_{i}}{3}\right\rceil \geq\left\lceil\frac{a_{1}}{3}-\frac{r}{3}+\sum_{i=1}^{r} \frac{a_{i}}{3}\right\rceil$ is equivalent to $a_{1}-(r-1)+\sum_{i=2}^{r}\left\lceil\frac{a_{i}}{3}\right\rceil \geq \frac{a_{1}}{3}-\frac{r}{3}+\sum_{i=1}^{r} \frac{a_{i}}{3}$ which holds precisely when $a_{1} \geq 2 r-3-\sum_{i=2}^{r} 3\left(\left\lceil\frac{a_{i}}{3}\right\rceil-\frac{a_{i}}{3}\right)$.

(iii) Since $a_{1}-(r-1)+\sum_{i=2}^{r}\left\lceil\frac{a_{i}}{3}\right\rceil$ is an integer, $a_{1}-(r-1)+\sum_{i=2}^{r}\left\lceil\frac{a_{i}}{r}\right\rceil \geq\left\lceil\frac{a_{1}+a_{2}+a_{3}}{2}\right\rceil-$ $1+\sum_{i=4}^{r}\left(\left\lceil\frac{a_{i}}{3}\right\rceil-1\right)$ is equivalent to $a_{1}+\left\lceil\frac{a_{2}}{3}\right\rceil+\left\lceil\frac{a_{2}}{3}\right\rceil \geq \frac{a_{1}+a_{2}+a_{3}}{2}+1$ which holds precisely when $a_{1} \geq 2+\left(a_{2}-2\left\lceil\frac{a_{2}}{3}\right\rceil\right)+\left(a_{3}-2\left\lceil\frac{a_{3}}{3}\right\rceil\right)$.

Note that if $a_{1} \geq 4$, we have $a_{1} \geq 2+2\left(a_{1}-2\left\lceil\frac{a_{1}}{3}\right\rceil\right) \geq 2+\left(a_{2}-2\left\lceil\frac{a_{2}}{3}\right\rceil\right)+\left(a_{3}-2\left\lceil\frac{a_{3}}{3}\right\rceil\right)$ where the last inequality holds since $a_{1} \geq a_{2} \geq a_{3}$.

Proof of Theorem 1.2 and Theorem 1.3. Let $r \geq 3$ and let $p_{1} \geq \cdots \geq p_{r} \geq 2$ (note that we already dealt with the case when $r=2$ in Corollary [3.6). Let $x_{1}, \ldots, x_{r}$ be integers with $0 \leq x_{i}<\frac{p_{i}}{3}$ for all $i \in[r]$. We have $p_{1} \geq \cdots \geq p_{r}$, but it might not be the case that $p_{1}-3 x_{1} \geq \cdots \geq p_{r}-3 x_{r}$, so we let $q_{1} \geq \cdots \geq q_{r}$ be integers and we let $\pi$ be a permutation on $[r]$ such that $p_{\pi(i)}-3 x_{\pi(i)}=q_{i}$ for all $i \in[r]$.

To prove Theorem 1.3 , we note that by Theorem 2.5 we have

$$
\begin{aligned}
R^{1 C}\left(p_{1}-3 x_{1}, \ldots, p_{r}-3 x_{r}\right) & \leq \max \left\{q_{1},\left\lceil\frac{q_{1}+q_{2}+q_{3}}{2}\right\rceil-1,\left\lceil\frac{q_{1}}{3}-\frac{r}{3}+\sum_{i=1}^{r} \frac{q_{i}}{3}\right\rceil\right\} \\
& \leq\left\lceil\frac{2 q_{1}}{3}-\frac{r}{3}+\sum_{i=1}^{r} \frac{q_{i}}{3}\right\rceil=\left\lceil\frac{2 p_{\pi(1)}}{3}-2 x_{\pi(1)}-\frac{r}{3}+\sum_{i=1}^{r}\left(\frac{p_{i}}{3}-x_{i}\right)\right\rceil .
\end{aligned}
$$

where the second inequality is by applying Fact 3.7.(i) (with $a_{i}=q_{i}$ ) to the second term in the maximum (the inequality is trivial for the other two terms in the maximum).

So we have

$$
R^{1 C}\left(p_{1}-3 x_{1}, \ldots, p_{r}-3 x_{r}\right)+\sum_{i=1}^{r} x_{i} \leq\left\lceil\frac{2 p_{\pi(1)}}{3}-2 x_{\pi(1)}-\frac{r}{3}+\sum_{i=1}^{r} \frac{p_{i}}{3}\right\rceil \leq\left\lceil p_{1}-\frac{r}{3}+\sum_{i=2}^{r} \frac{p_{i}}{3}\right\rceil,
$$

and thus by Theorem 3.3, $R^{P M}\left(p_{1}, \ldots, p_{r}\right)=f^{3}\left(p_{1}, \ldots, p_{r}\right) \leq\left\lceil p_{1}-\frac{r}{3}+\sum_{i=2}^{r} \frac{p_{i}}{3}\right\rceil$ as desired.

Next we prove Theorem 1.2. The lower bound follows from Corollary [3.5.(i). For the upper bound, we set $m:=\max \left\{q_{1},\left\lceil\frac{q_{1}+q_{2}+q_{3}}{2}\right\rceil-1,\left\lceil\frac{q_{1}}{3}-\frac{r}{3}+\sum_{i=1}^{r} \frac{q_{i}}{3}\right\rceil\right\}$ and note that by Theorem 2.5. we have

$$
\begin{aligned}
& R^{1 C}\left(p_{1}-3 x_{1}, \ldots, p_{r}-3 x_{r}\right)+\sum_{i=1}^{r} x_{i} \leq m+\sum_{i=1}^{r} x_{i} \\
& \stackrel{*}{\leq} \max \left\{p_{1}-(r-1)+\sum_{i=2}^{r}\left\lceil\frac{p_{i}}{3}\right\rceil,\left\lceil\frac{p_{1}+p_{2}+p_{3}}{2}\right\rceil-1+\sum_{i=4}^{r}\left(\left\lceil\frac{p_{i}}{3}\right\rceil-1\right),\left\lceil\frac{p_{1}}{3}-\frac{r}{3}+\sum_{i=1}^{r} \frac{p_{i}}{3}\right\rceil\right\} \\
& \leq p_{1}-(r-1)+\sum_{i=2}^{r}\left\lceil\frac{p_{i}}{3}\right\rceil,
\end{aligned}
$$

where the last inequality follows by Fact 3.7.(ii) and Fact 3.7.(iii) (with $a_{i}=p_{i}$ ). It remains to justify the inequality $\stackrel{*}{\leq}$ above.

Case $1\left(m=q_{1}\right)$ Set $J=[r] \backslash\{\pi(1)\}$. We have

$$
\begin{aligned}
R^{1 C}\left(p_{1}-3 x_{1}, \ldots, p_{r}-3 x_{r}\right)+\sum_{i=1}^{r} x_{i} \leq p_{\pi(1)}-2 x_{\pi(1)}+\sum_{j \in J} x_{j} & \leq p_{\pi(1)}-2 x_{\pi(1)}+\sum_{j \in J}\left(\left\lceil\frac{p_{j}}{3}\right\rceil-1\right) \\
& \leq p_{1}-(r-1)+\sum_{i=2}^{r}\left\lceil\frac{p_{i}}{3}\right\rceil .
\end{aligned}
$$


Case $2\left(m=\left\lceil\frac{q_{1}+q_{2}+q_{3}}{2}\right\rceil-1\right)$ Set $J=[r] \backslash\{\pi(1), \pi(2), \pi(3)\}$. We have

$$
\begin{aligned}
R^{1 C}\left(p_{1}-3 x_{1}, \ldots, p_{r}-3 x_{r}\right)+\sum_{i=1}^{r} x_{i} & \leq\left\lceil\frac{p_{\pi(1)}-x_{\pi(1)}+p_{\pi(2)}-x_{\pi(2)}+p_{\pi(3)}-x_{\pi(3)}}{2}\right\rceil-1+\sum_{j \in J} x_{j} \\
& \leq\left\lceil\frac{p_{\pi(1)}+p_{\pi(2)}+p_{\pi(3)}}{2}\right\rceil-1+\sum_{j \in J}\left(\left\lceil\frac{p_{j}}{3}\right\rceil-1\right) \\
& \leq\left\lceil\frac{p_{1}+p_{2}+p_{3}}{2}\right\rceil-1+\sum_{i=4}^{r}\left(\left\lceil\frac{p_{i}}{3}\right\rceil-1\right) .
\end{aligned}
$$

Case $3\left(m=\left\lceil\frac{q_{1}}{3}-\frac{r}{3}+\sum_{i=1}^{r} \frac{q_{i}}{3}\right\rceil\right)$ We have

$$
R^{1 C}\left(p_{1}-3 x_{1}, \ldots, p_{r}-3 x_{r}\right)+\sum_{i=1}^{r} x_{i} \leq\left\lceil\frac{p_{\pi(1)}}{3}-x_{\pi(1)}-\frac{r}{3}+\sum_{i=1}^{r} \frac{p_{i}}{3}\right\rceil \leq\left\lceil\frac{p_{1}}{3}-\frac{r}{3}+\sum_{i=1}^{r} \frac{p_{i}}{3}\right\rceil .
$$

\subsection{Small values of $p_{i}$ and small number of colors}

We now determine the Ramsey number of path-matchings for at most 4 colors.

\section{Corollary 3.8.}

(i) For all integers $p_{1} \geq p_{2} \geq 2, R^{P M}\left(p_{1}, p_{2}\right)=p_{1}+\left\lceil\frac{p_{2}}{3}\right\rceil-1$.

(ii) For all integers $p_{1} \geq p_{2} \geq p_{3} \geq 2$ such that $\left(p_{1}, p_{2}, p_{3}\right) \neq(3,3,3), R^{P M}\left(p_{1}, p_{2}, p_{3}\right)=$ $p_{1}+\left\lceil\frac{p_{2}}{3}\right\rceil+\left\lceil\frac{p_{3}}{3}\right\rceil-2$. Furthermore $R^{P M}(3,3,3)=4$.

(iii) For all integers $p_{1} \geq p_{2} \geq p_{3} \geq p_{4} \geq 2$ such that $\left(p_{1}, p_{2}, p_{3}, p_{4}\right) \notin\{(3,3,3,3),(4,3,3,3)\}$, $R^{P M}\left(p_{1}, p_{2}, p_{3}, p_{4}\right)=p_{1}+\left\lceil\frac{p_{2}}{3}\right\rceil+\left\lceil\frac{p_{3}}{3}\right\rceil+\left\lceil\frac{p_{4}}{3}\right\rceil-3$. Furthermore $R^{P M}(3,3,3,3)=4$ and $R^{P M}(4,3,3,3)=5$.

Proof. Note that by Observation 2.1, we may assume that $p_{1} \geq p_{2} \geq p_{3} \geq p_{4} \geq 3$.

(i), (ii) If $p_{1} \geq 4$, we may apply Theorem 1.2 Otherwise $p_{1} \leq 3$ in which case we are done by Observation 2.2

(iii) If $p_{1} \geq 5=2 \cdot 4-3$, then we may apply Theorem 1.2 . So suppose $p_{1} \leq 4$. If $\left(p_{1}, p_{2}, p_{3}, p_{4}\right) \in$ $\{(4,4,4,4),(4,4,4,3),(4,4,3,3)\}$, then we may still apply Theorem[1.2 If $p_{1}=p_{2}=p_{3}=p_{4}=3$, then we may apply Observation 2.2. So we only have to check the case where $\left(p_{1}, p_{2}, p_{3}, p_{4}\right)=$ $(4,3,3,3)$ which is easily seen by direct inspection.

Scobee [16] determined the Ramsey number $R\left(m_{1} P_{3}, m_{2} P_{3}, m_{3} P_{3}\right)=m_{2}+m_{3}+3 m_{1}-2$ for $m_{1} \geq m_{2} \geq m_{3}$ and $m_{1} \geq 2$ with a difficult (20 page) proof. This implies Corollary 3.8 (ii) when all $p_{i}$ 's are divisible by 3 .

We now prove a result about $R_{r}^{P M}(p)$ when $p=4,5$ (we already determined $R_{r}^{P M}(3)$ in Observation 2.2). To do so, we first establish the following.

Proposition 3.9. For all integers $r \geq 2, R_{r}^{1 C}(4) \leq r+3, R_{r}^{1 C}(5) \leq r+4$, and $R_{r}^{1 C}(6) \leq r+5$. $r$.

Recall that in the language of covering designs, we have $R_{r}^{1 C}(p) \leq v$ if and only if $C(v, p-1)>$

Proof. We have $C(r+3,3) \geq \frac{(r+3)(r+2)}{3 \cdot 2}>r$ for all $r$, and thus $R_{r}^{1 C}(4) \leq r+3$. We have $C(r+4,4) \geq \frac{(r+4)(r+3)}{4 \cdot 3}>r$ for all $r$, and thus $R_{r}^{1 C}(5) \leq r+4$. Finally, we have $C(r+$ $5,5) \geq\left\lceil\frac{r+4}{4}\left\lceil\frac{r+3}{3}\right\rceil\right\rceil>r$ for all $r$ such that $r \notin\{4,8\}$. However, in the case when $r=4$ and $r=8$, it is known (see [13] for instance) that $C(9,5)=5>4$ and $C(13,5)=10>8$. Thus $R_{r}^{1 C}(6) \leq r+5$. 
Proposition 3.10. For all $r \geq 2, R_{r}^{P M}(4)=r+3$ and $R_{r}^{P M}(5)=r+4$.

Proof. By Proposition 3.9 and Theorem 3.3, we have

$$
R_{r}^{P M}(4)=\max \left\{R_{s}^{1 C}(4)+r-s: s \in[r]\right\}=\max \{s+3+r-s: s \in[r]\}=r+3
$$

and

$$
R_{r}^{P M}(5)=\max \left\{R_{s}^{1 C}(5)+r-s: s \in[r]\right\}=\max \{s+4+r-s: s \in[r]\}=r+4 .
$$

\section{Conclusion}

We have determined $R^{P M}\left(p_{1}, \ldots, p_{r}\right)$ exactly unless $p_{1} \leq 2 r-4-\sum_{i=2}^{r} 3\left(\left\lceil\frac{p_{i}}{3}\right\rceil-\frac{p_{i}}{3}\right)$ and we have determined $R^{P M}\left(p_{1}, \ldots, p_{r}\right)$ to within a constant (depending on $r$ ) in every case. It would certainly be interesting to solve these remaining cases exactly, although Corollary 3.5 gives some evidence that this remaining case where $p_{1}$ is fixed and $r$ is large could be challenging.

In particular, we think the following problem is worth focusing on.

Problem 4.1. For all integers $r \geq 5$ and $p \geq 6$ such that $p \equiv 0 \bmod 3$ or $p \equiv 2 \bmod 3$, determine $R_{r}^{P M}(p)$ exactly.

Note that the case when $r \leq 4$ follows from Observation 3.8. The case when $p=2$ is trivial, the case $p=3$ is Observation 2.2, the cases $p=4,5$ follow from Proposition 3.10, and the case $p \equiv 1$ mod 3 follows from Theorem 1.2. Also note that by Theorem 1.2 we may assume $r \geq \frac{p+4}{2}$ when $p \equiv 0 \bmod 3$ and we may assume $r \geq p+2$ when $p \equiv 2 \bmod 3$.

It already seems non-trivial to determine $R_{r}^{P M}(6)$ for all $r \geq 5$, because in order to do so, one needs to compute (or appropriately bound) $R^{1 C}\left(p_{1}, \ldots, p_{a}, p_{a+1}, \ldots, p_{r}\right)$ where $p_{1}=\cdots=p_{a}=6$ and $p_{a+1}=\cdots=p_{r}=3$ for all $0 \leq a \leq r$.

Another question relates to our estimates on $R^{1 C}\left(p_{1}, \ldots, p_{r}\right)$. We think of $p_{1}-(r-1)+$ $\sum_{i=2}^{r}\left\lceil\frac{p_{i}}{3}\right\rceil$ as being the "standard" value of $R^{P M}\left(p_{1}, \ldots, p_{r}\right)$, but of course we know there are examples where $R^{P M}\left(p_{1}, \ldots, p_{r}\right)>p_{1}-(r-1)+\sum_{i=2}^{r}\left\lceil\frac{p_{i}}{3}\right\rceil$. However, all such examples stem from the fact that $R_{s}^{1 C}(3)=\left|\frac{\sqrt{8 s+1}+1}{2}\right|+1$ (see Corollary 3.5). In every other case we know of, we have $R^{1 C}\left(p_{1}, \ldots, p_{r}\right) \leq p_{1}-(r-1)+\sum_{i=2}^{r}\left\lceil\frac{p_{i}}{3}\right\rceil$ (and in many cases it is much smaller). So this raises the following question.

Question 4.2. Under what circumstances do we have $R^{1 C}\left(p_{1}, \ldots, p_{r}\right) \leq p_{1}-(r-1)+\sum_{i=2}^{r}\left\lceil\frac{p_{i}}{3}\right\rceil$ ? Is this always true when none of $p_{1} \geq \cdots \geq p_{r}$ are divisible by 3? Is this always true when $p_{1} \geq \cdots \geq p_{r} \geq 4$ ?

One case in which we are confident the above is true is when $p=p_{1}=\cdots=p_{r} \geq 4$ (and we proved it for $4 \leq p \leq 6$ in Proposition 3.9. .

Another reason it seems to be difficult to determine $R^{P M}\left(p_{1}, \ldots, p_{r}\right)$ exactly in all cases is that the lower bound examples are not necessarily unique. We have multiple examples where $R^{P M}\left(p_{1}, \ldots, p_{r}\right)=R^{1 C}\left(p_{1}, \ldots, p_{r}\right)$ and in those cases the lower bound for $R^{P M}\left(p_{1}, \ldots, p_{r}\right)$ comes from the extremal coloring $\left[p_{1}-1,\left\lceil\frac{p_{2}}{3}\right\rceil-1, \ldots,\left\lceil\frac{p_{r}}{3}\right\rceil-1\right]$ whereas the lower bound for $R^{1 C}\left(p_{1}, \ldots, p_{r}\right)$ comes from a covering design. For instance, we have $R^{P M}(5,5,5)=7=$ $R^{1 C}(5,5,5)$. The extremal coloring $[4,1,1]$ provides a lower bound for $R^{P M}(5,5,5)$, whereas a lower bound for $R^{1 C}(5,5,5)$ comes from partitioning the vertices of $K_{6}$ into $A_{1}, A_{2}, A_{3}$ with $\left|A_{i}\right|=2$ and coloring edges in $A_{i}$ and in $\left[A_{i}, A_{i+1}\right]$ with color $i$.

Another example is $R^{P M}(4,3,3,3)=5=R^{1 C}(4,3,3,3)$. Coloring the edges of a $K_{4}$ by coloring a triangle with colors $2,3,4$ and coloring the other edges with color 1 provides a lower bound for $R^{P M}(4,3,3,3)$; whereas coloring a triangle with color 1 and coloring the other edges with three different colors provides the lower bound for $R^{1 C}(4,3,3,3)>4$.

On the other hand we have many examples where $R^{P M}\left(p_{1}, \ldots, p_{r}\right)>R^{1 C}\left(p_{1}, \ldots, p_{r}\right)$, such as $R^{P M}(4,4,4)=6>5=R^{1 C}(4,4,4)$. So this raises the following question. 
Question 4.3. Under what circumstances do we have $R^{P M}\left(p_{1}, \ldots, p_{r}\right)=R^{1 C}\left(p_{1}, \ldots, p_{r}\right)$ ?

Acknowledgement. Thanks to André E. Kezdy for his help to obtain the thesis [16] and to Jácint Szabó for helping with the reference [1]. We also thank the referee for some helpful remarks.

\section{References}

[1] A. Binkle, Structural results on maximal $k$-degenerate graphs, Discussiones Mathematicae Graph Theory, 32 (2012), pp. 659-676.

[2] B. Bollobás. Random graphs, Cambridge university press, no 73 (2001).

[3] S.A. Burr and J. A. Roberts. On Ramsey numbers for linear forests. Discrete Mathematics, 8, no. 3 (1974), pp. 245-250.

[4] E. J. Cockayne, P. J. Lorimer, The Ramsey number for stripes, J. Austral. Math. Soc., 19 (1975), pp. 252-256.

[5] P. Erdős, H. Hanani, On a limit theorem in combinatorial analysis, Publ. Math. Debrecen, 10 (1963), pp. 10-13.

[6] R.J. Faudree and R.H. Schelp, Ramsey numbers for all linear forests, Discrete Mathematics, 16 (1976), pp. 149-165.

[7] Z. Füredi, Matchings and covers in hypergraphs, Graphs and Combinatorics, 4 (1988), pp. 115-206.

[8] L. Gerencsér and A. Gyárfás, On Ramsey-Type Problems, Annales Universitatis Scientiarum Budapestiensis, Eötvös, Sect. Math., 10 (1967), pp. 167-170.

[9] A. Gyárfás, Large monochromatic components in edge colorings of graphs: A survey, in: Ramsey Theory, Birkhäuser, 2011, A. Soifer editor, Progress in Mathematics, vol. 285, pp. 77-96.

[10] P. Horák and N. Sauer, Covering complete graphs by cliques, Ars Combinatoria 33 (1992), pp. 279-288.

[11] M. Las Vergnas, An extension of Tutte's 1-factor theorem, Discrete Mathematics, 23 (1978), pp. 241-255.

[12] L. Lovász, M. Plummer, Matching Theory, Budapest, Akadémiai Kiadó (1986).

[13] W. H. Mills, Covering designs I: Covering by a small number of subsets, Ars Combinatoria, 8 (1979), pp. 199-315.

[14] F. Petrov, A direct proof that every $r$-colored complete graph on $n=(r+1) m-(r-1)$ vertices has a monochromatic matching of size m, https://mathoverflow.net/questions/299423/

[15] J. Schönheim, On coverings, Pacific Journal of Mathematics, 14 (1964) pp. 1405-1411.

[16] M. W. Scobee, On the Ramsey number $r\left(m_{1} P_{3}, m_{2} P_{3}, m_{3} P_{3}\right)$ and related results, $M A$ thesis, University of Louisville (1993).

[17] S. B. Seidman, Network structure and minimum degree, Social networks, 5 (1983), pp. 269-287.

[18] R. M. Wilson, An existence theory for pairwise balanced designs I-III, Journal of Combinatorial Theory A, 13 (1972) pp. 220-273, 18 (1975) pp. 71-79.

[19] C. Xu, H. Yang, S. Zhang, A new proof on the Ramsey number of matchings, arXiv:1905.08456v1 